\title{
The utility of FDG-PET in the diagnosis of thymic epithelial tumors
}

\author{
Hideki Otsuka, MD,PhD \\ Department of Medical Imaging, Institute of Health Biosciences, Tokushima University Graduate School, \\ Tokushima, Japan
}

\begin{abstract}
In this article, seven studies evaluating the utility of FDG-PET in the diagnosis of thymic epithelial tumors were reviewed and the results of an investigation conducted in Tokushima University Hospital are reported. FDG accumulation of low-risk thymoma is low, and FDG accumulation of thymic carcinoma is high. High-risk thymoma exhibits various degrees of accumulation and a definite conclusion has not yet been reached ; however, overall, the higher the histological grade, the higher the FDG accumulation becomes, suggesting that this is correlated to staging. Our data also support the previous reports. FDG-PET may indicate lymph node metastasis and distant metastasis, which are sometimes difficult to detect using other modalities. When lymph node metastasis and/or distant metastasis are suspected in addition to thymic lesions, FDG-PET/CT, which allows for searching of the entire body at once, is useful. FDG-PET/CT is effective for estimating histopathological malignancy and staging in the diagnosis of thymic epithelial tumors and can be an important imaging test with high relevance to the prognosis of a patient. J. Med. Invest. 59 : 225-234, August, 2012
\end{abstract}

Keywords : thymic epithelial tumor, FDG-PET, diagnosis, staging, pathology

\section{INTRODUCTION}

Various neoplasms, including thymus-related lesions, lymphomas and germ cell tumors, involve the anterior mediastinum. Thymic epithelial tumors are the most common type of thymus-related lesions. Thymic epithelial tumors are classified in two major groups, thymomas and thymic carcinomas, in the latest World Health Organization (WHO) classification (2004). There are various pathological types of thymic epithelial tumors, and the clinical features and prognosis associated with each tumor depend on the pathological type. Furthermore, the WHO histological classification and Masaoka staging, a surgical staging system based on the presence of

Received for publication July 2, 2012 ; accepted July 20, 2012.

Address correspondence and reprint requests to Hideki Otsuka, $\mathrm{MD}, \mathrm{PhD}$, Kuramoto-cho 3-18-15, Toksuhima city, Tokushima, Japan 770-8503 and Fax : +81-88-633-7174. invasion in the surrounding organs, closely correlate with prognosis. To differentiate and predict the histology and initial staging of tumors, conducting less invasive imaging evaluations prior to administering any invasive procedures or treatments is important. CT and MRI are useful for evaluating the morphological appearance of tumors and the presence of invasion in surrounding fat tissues or structures such as the pleura, lungs or vessels. However, some findings are overlapping, which thus makes it difficult to diagnose histological subtypes correctly. On the other hand, fluoro-deoxy-glucose positron emission tomography (FDG-PET) can provide useful metabolic information regarding thymic lesions.

In this article, the available literature on the utility of FDG-PET in the diagnosis of thymic epithelial tumors is reviewed and the results of an investigation conducted in our institute are reported. 


\section{THYMIC EPITHELIAL TUMORS}

\section{Histological classification (Table 1)}

The World Health Organization (WHO) published the histological classification in 1999. In this version, thymomas were divided into five subtypes and thymic carcinomas were classified as type $\mathrm{C}$. The WHO classification was renewed in 2004 and this version of the classification is widely used today. In the 2004 classification, rare subtypes were added to traditional types, type $\mathrm{C}$ was removed and thymic carcinomas were classified in various subtypes, including neuroendocrine epithelial tumors. Thymomas were divided into type A and type B according to the shape of the tumor cells and nuclei. Type B was also subdivided in three subtypes, B1, B2 and B3, according to the number of lymphocytes and the atypia of the tumor cells. Type B1 is lymphocytedominant and type B3 is epithelial componentdominant. The grade of malignancy increases with the progression of each subtype: $A, A B, B 1, B 2$, B3. Thymic carcinomas were defined as all malignant non-organotypic epithelial neoplasms except germ cell tumors. Squamous cell carcinomas are the most common type of thymic carcinomas. Thymic epithelial tumors are divided into three subgroups based on prognosis and WHO histological classification : low-risk (LR), including types $\mathrm{A}, \mathrm{AB}$ and $\mathrm{B} 1$; high-risk (HR), including types B2 and B3 ; and thymic carcinomas (CA).

\section{Clinical staging}

Masaoka staging is widely used to assess thymomas (Table 2) and is closely correlated with the prognosis. Masaoka staging is based on the presence of invasion in the capsule or surrounding organs, the existence of pleural or pericardial dissemination and the presence of lymphogenous or hematogenous metastasis. WHO TNM classification for malignant thymic epithelial tumors was published in 2009 (Table 3).

\section{FDG-PET}

Review of previous reports

The author searched the PubMed database inserting the following key words : "thymus," "epithelial," "tumor," "PET" and "FDG" and found seven studies evaluating the utility of FDG-PET in the diagnosis of thymic epithelial tumors. The author also added the results of an investigation conducted in

Table 1 : WHO histological classification

\begin{tabular}{cll}
\hline Type & Risk & \multicolumn{1}{c}{ Description } \\
\hline Type A & Low & $\begin{array}{l}\text { Composed of a homogeneous population of neoplastic eplthelial cells having spindle and oval } \\
\text { shapes, lacking nuclear atypia, and accompanied by few or no non-neoplastic lymphocytes }\end{array}$ \\
Type AB & $\begin{array}{l}\text { Foci have the features of type A thymoma and are admixed with foci rich inl ymphocytes, with } \\
\text { sharp or indistinct segregation of the two patterns } \\
\text { Resembles the normal functional thymus in that it combines large expanses with an appearance } \\
\text { practically indistinguishable from normal thymic cortex with areas resembling thymic medulla }\end{array}$ \\
Type B1 & $\begin{array}{l}\text { Neoplastic epithelial component appears as scattered plump cells with vesicular nuclei and } \\
\text { distinct nucleoli among a predominant population of lymphocytes ; perivascular spaces are } \\
\text { common } \\
\text { Type B2 }\end{array}$ & $\begin{array}{l}\text { Predominantly composed of epithelial cells with a round or polygonal shape and exhibiting no } \\
\text { or mild atypia, admixed with a minor component of lymphocytes ; foci of squamous metaplasia } \\
\text { and perivascular spaces are common }\end{array}$ \\
\hline Type B3 & $\begin{array}{l}\text { Exhibits clear-cut cytologic atypia and a set of cytoarchitectural features no longer specific to } \\
\text { thymus but, rather, analogous to those seen in carcinomas of other organs }\end{array}$ \\
\hline Thymic carcinoma &
\end{tabular}

Table 2 : Masaoka staging system

\begin{tabular}{cl}
\hline Stage & Description \\
\hline I & Encapsulated tumor without microscopic evidence of capsular invasion \\
II & Microscopic or macroscopic invasion of the surounding fatty tissue or the mediastinal pleura \\
III & Macroscopic invasion of neighboring organs, such as the pericardium, lung, or the great vessels \\
IVa & Pericardial or pleural dissemination \\
IVb & Lymph node or distant metastasis \\
\hline
\end{tabular}


Table 3 : TNM classification of Thymic Epithelial Tumors (WHO)

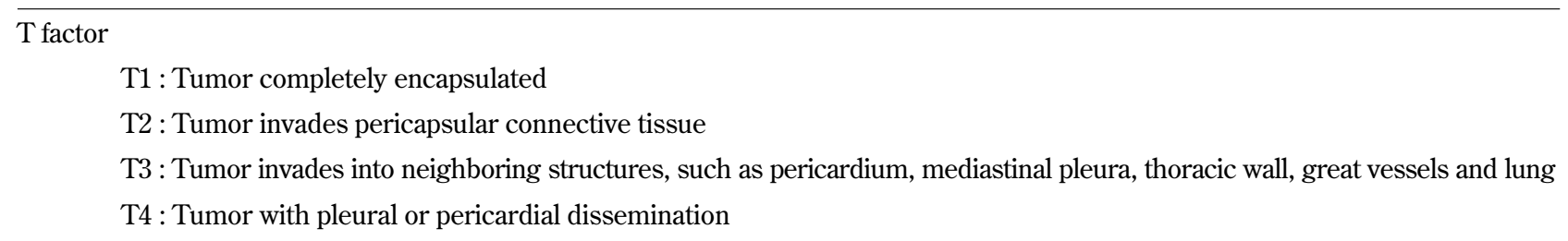

$\mathrm{N}$ factor

N0 : No lymph node metastasis

$\mathrm{N} 1$ : Metastasis to anterior mediastinal lymph nodes

N2 : Metastasis in other intrathoracic lymph nodes excluding anterior mediastinal lymph nodes

N3 : Metastasis in scalene and/or supraclavicular lymph nodes

M factor

M0 : No distant organ metastasis

M1 : Distant organ metastasis

$\begin{array}{ll}\text { Stage I } & \text { T1 N0 MO } \\ \text { Stage II } & \text { T2 N0 M0 } \\ \text { Stage III } & \text { T1 N1 M0 } \\ & \text { T2 N1 M0 } \\ & \text { T3 N0, 1 M0 } \\ \text { Stage IV } & \text { T4 Any N M0 } \\ & \text { Any T N2, 3 M0 } \\ & \text { Any T Any N M1 }\end{array}$

Tokushima University Hospital to this article.

A summary is shown in Table 4 (1-7). Three of the seven articles grouped thymic epithelial tumors into three subgroups (low-risk, high-risk and thymic carcinomas) $(1,2,4)$, three articles classified thymic epithelial tumors into two subgroups (lowrisk and high-risk (types B2, B3 and thymic carcinomas) $(3,5,7)$ and one article grouped thymic epithelial tumors into thymoma and thymic carcinoma groups (6). Two articles used the $\mathrm{T} / \mathrm{M}$ ratio of maximum standardized uptake value (SUVmax) to evaluate the degree of FDG uptake $(2,4)$. One article reported the use of dual time-point, early and delayed scans (3). Two articles mentioned the biologic correlations between thymic epithelial tumors and glucose transporters (Glut), hexokinase, hypoxia, angiogenesis and cell cycle regulators $(4,5)$. Sung et al. classified 33 cases of thymic epithelial tumors into low-risk (types A, AB and B1), highrisk (types B2 and B3) thymoma, and thymic carcinoma, and investigated the FDG-PET/CT as well as enhanced CT. The SUVmax of the low-risk and high-risk groups was statistically significantly lower than that of thymic carcinoma. FDG uptake pattern and mediastinal fat invasion using enhanced CT was useful for differentiating subgroups ; whereby, it was determined that lymph node metastasis that could not be indicated using enhanced CT was indicated using PET/CT in 2 of 33 patients, and it was reported that PET/CT was useful for differentiating subgroups as well as the staging of thymic epithelial tumors (1). Endo et al. divided 36 cases of thymic epithelial tumors into 3 groups (low-risk, highrisk and thymic carcinoma) in the same manner as Sung et al., and the SUVmax of the lesions with respect to the mediastinal SUVmax was investigated as a T/M ratio (ratio of the peak SUV of the tumor to the mean SUV of the mediastinum at the level of the aortic arch). As a result, it was reported that 3 groups may be differentiating using the $\mathrm{T} / \mathrm{M}$ ratio, and that PET was useful in the evaluation of thymic epithelial tumors (2). Kaira et al. also evaluated thymic epithelial tumors in 49 patients using the $\mathrm{T} / \mathrm{M}$ ratio, reporting that the $T / M$ ratio was significantly high in relation to the grade of malignancy. In this paper, it is shown that the biologic mechanism of the FDG uptake to thymic epithelial tumors is related to glucose metabolism, hypoxia, angiogenesis 
Table 4 : A summary of seven articles. LR : Low-risk, HR : High-risk, CA : carcinoma, SUVmax : maximum standardized uptake value

\begin{tabular}{|c|c|c|c|c|c|c|c|c|}
\hline Author & Year & No. of patients & $\mathrm{M} / \mathrm{F}$ & $\begin{array}{l}\text { year mean } \\
\text { (range) }\end{array}$ & group & number & Parameter & Statistic analysis \\
\hline \multirow[t]{4}{*}{ Sung et al (1) } & 2006 & 33 & $15 / 18$ & $55(34-68)$ & & & $\underline{\text { SUVmax }}$ & \\
\hline & & & & & LR & 8 & $4.0(0.42)$ & LR vs HR: $p=0.076$ \\
\hline & & & & & HR & 9 & $5.6(1.90)$ & LR vs CA: $p<0.001$ \\
\hline & & & & & $\mathrm{CA}$ & 16 & $10.5(4.68)$ & HR vs CA: $p<0.001$ \\
\hline \multirow[t]{4}{*}{ Endo et al (2) } & 2008 & 36 & $21 / 15$ & $59(32-80)$ & & & $\underline{\mathrm{T} / \mathrm{M} \text { ratio }}$ & \\
\hline & & & & & LR & 15 & $2.64(0.78)$ & LR vs HR: $p=0.01$ \\
\hline & & & & & HR & 10 & $4.29(1.41)$ & HR vs $\mathrm{CA}: p=0.01$ \\
\hline & & & & & $\mathrm{CA}$ & 11 & $8.90(3.62)$ & \\
\hline \multirow[t]{8}{*}{ Inoue et al (3) } & 2009 & 46 & $29 / 17$ & $58(31-75)$ & & & early SUVmax & \\
\hline & & & & & LR & 23 & 3.2 & HR vs LR: $p<0.001$ \\
\hline & & & & & HR & 23 & 6 & \\
\hline & & & & & (B2, B3) & 12 & & \\
\hline & & & & & (CA) & 11 & & \\
\hline & & & & & & & delayed SUVmax & \\
\hline & & & & & LR & & 3.4 & HR vs LR: $p=0.001$ \\
\hline & & & & & HR & & 7.4 & \\
\hline \multirow[t]{4}{*}{ Kaira et al (4) } & 2010 & 49 & $23 / 26$ & $64(32-80)$ & & & $\underline{\mathrm{T} / \mathrm{M} \text { ratio }}$ & \\
\hline & & & & & LR & 27 & $2.6(0.9)$ & LR vs HR: $p=0.002$ \\
\hline & & & & & HR & 11 & $4.3(1.6)$ & HR vs CA: $p=0.008$ \\
\hline & & & & & $\mathrm{CA}$ & 11 & $8.9(3.6)$ & \\
\hline \multirow[t]{5}{*}{ Nakajo et al (5) } & 2010 & 11 & $5 / 6$ & $55(41-71)$ & & & $\underline{\text { SUVmax }}$ & \\
\hline & & & & & LR & 6 & $3.05(0.55)$ & LR vs HR: $p=0.008$ \\
\hline & & & & & $\mathrm{HR}$ & 5 & $5.24(2.44)$ & \\
\hline & & & & & (B2, B3) & 4 & & \\
\hline & & & & & (CA) & 1 & & \\
\hline \multirow[t]{5}{*}{ Igai et al (6) } & 2010 & 13 & $6 / 7$ & $59(36-78)$ & & & $\underline{\text { SUVmax }}$ & \\
\hline & & & & & Thymoma & 8 & $3.43(2.19)$ & Thymoma vs CA: $p=0.002$ \\
\hline & & & & & LR & 3 & & \\
\hline & & & & & $\mathrm{HR}$ & 5 & & \\
\hline & & & & & $\mathrm{CA}$ & 5 & $8.15(7.88)$ & \\
\hline \multirow[t]{5}{*}{ Terzi et al (7) } & 2011 & 26 & $14 / 12$ & $56(34-85)$ & & & $\underline{\text { SUVmax }}$ & \\
\hline & & & & & LR & 14 & $4.0(1.7)$ & LR vs HR: $p<0.01$ \\
\hline & & & & & HR & 12 & $14.1(8.3)$ & \\
\hline & & & & & (B2, B3) & 4 & & \\
\hline & & & & & (CA) & 8 & $17.1(8.5)$ & \\
\hline \multirow[t]{5}{*}{ Otsuka } & 2012 & 35 & $17 / 18$ & $62(27-84)$ & & & $\underline{\text { SUVmax }}$ & \\
\hline & & & & & Thymoma & 24 & $3.9(2.0)$ & LR vs CA ; $p=0.007$ \\
\hline & & & & & LR & 11 & $3.7(1.8)$ & HR vs CA: $p=0.008$ \\
\hline & & & & & HR & 13 & $4.0(2.8)$ & LR vs HR: $p=0.99$ \\
\hline & & & & & $\mathrm{CA}$ & 11 & $7.6(3.7)$ & Thymoma vs $\mathrm{CA}: p=0.0004$ \\
\hline
\end{tabular}


and cell cycle regulator (4). Inoue et al. classified 46 cases into low risk tumors regarding type A, $\mathrm{AB}$, and $\mathrm{B} 1$ and high-risk tumors regarding type $\mathrm{B} 1,2$ and thymic carcinoma, and reported the SUVmax to be significantly higher in high risk tumors. It has been determined that high risk and low risk tumors may be differentiated with the highest precision when the cutoff value of SUVmax is set at 4.5. Furthermore, in addition to the normal imaging following an hour (early SUVmax), a delayed scan was conducted after 3 hours and the rate of variability of SUVmax with delayed SUVmax, early scan, and delayed scan were calculated as the retention index (RI). Regardless of the type of tumor, a higher value was shown in delayed SUVmax than early SUVmax. It is reported that there was no significant difference in RI between the low risk group and the high risk group (3). Nakajo et al. classified 11 patients into low risk tumors regarding type $\mathrm{A}, \mathrm{AB}$, and $\mathrm{B} 1$ and high-risk tumors regarding type B2, 3 and thymic carcinoma, and determined that SUVmax exhibited a significantly higher value in high risk than low risk tumors. Furthermore, it is reported that FDG accumulation was highly correlated to Glut-1 and hexokinase II which promotes glycolysis (5). Terzi et al. divided thymic epithelial tumors into 2 groups of low risk and high risk, and reported that there is a strong correlation between SUVmax and the subgroup, as well as between SUVmax and staging. Moreover, it has been determined that low risk and high risk cases may be differentiated when the $\mathrm{T} / \mathrm{M}$ ratio $=2.75$ is set as the cut-off value (7). Igai et al. divided 13 patients into the thymoma group and the thymic carcinoma group, reporting that SUVmax was high in the thymic carcinoma group among the two groups with a statistical difference, and that FDG-PET was useful in predicting the malignancy grade of the WHO (6).

\section{INVESTIGATION IN TOKUSHIMA UNIVER- SITY HOSPITAL}

\section{Patients and methods}

This retrospective study was approved by the institutional review board of Tokushima University Hospital. The research content was posted on the information board at the entrance to the Imaging Center of Tokushima University Hospital. Written informed consent for FDG-PET/CT examination and contrast enhanced CT was obtained from all patients.

\section{Patient population}

Thirty-five (17 males, 18 females ; age range 27 to 84 years, mean/SD :61.6/14.3) patients with FDG-positive and pathologically confirmed thymic epithelial lesions who underwent FDG-PET/CT and enhanced CT or non-enhanced diagnostic $\mathrm{CT}$ prior to initial surgery, chemotherapy or radiation therapy were enrolled in this study.

\section{FDG-PET/CT}

All FDG-PET/CT examinations were performed before any treatments were administered. All patients were instructed to be fast for at least 6 hours before FDG injection. The FDG-PET/CT images were obtained one hour after the intravenous injection of $3.7 \mathrm{MBq} / \mathrm{kg}$ body weight of FDG. The patients' blood glucose levels were confirmed to be less than $150 \mathrm{mg} / \mathrm{dl}$ immediately prior to the administration of FDG. Images were obtained using a hybrid PET/CT scanner (Aquiduo, Toshiba, Japan) in 3-dimensional mode under free breathing. The first images were localized images taken from the top of the skull to the middle of the thigh followed by CT images obtained with a 16 -slice multidetector CT system. Emission data were acquired in the caudo-cranial direction from the middle of the thigh to the top of the skull. Two minutes of acquisition time were used per bed position and most patients required eight bed positions. Image reconstruction was completed using an ordered subset expectation maximization (OSEM) algorithm (two iterations, 14 subsets). Attenuation-corrected emission data were obtained using filtered back projection CT data.

\section{Chest CT}

CT scans were performed primarily at Tokushima University Hospital (32 patients), with the exception of three patients. Isovoxel data were acquired using 16 -slice multi-detector CT performed in our institution with $1 \mathrm{~mm}$ slice thickness and $2 \mathrm{ml} / \mathrm{kg}$ body weight of iodine contrast material. Thirty enhanced and five non-enhanced CT images were reviewed. Scanning protocol such as scanning time and the injection rate, dose and iodine concentration of the contrast material were varied according to patient condition, including patient body weight and renal function.

\section{Image interpretation}

Two observers (board certificated physicians of 
diagnostic radiology/nuclear medicine/PET and diagnostic radiology/PET) reviewed the FDG-PET/ $\mathrm{CT}$, enhanced and non-enhanced chest CT images. The chest CT, PET and CT portions of the PET/ CT images were analyzed for attenuation correction and anatomical recognition, and the fused PET/CT images obtained in the axial, transverse and coronal views were interpreted using Aquarias Net software (TeraRecon). The maximum standardized uptake value (SUVmax) of each lesion was measured to evaluate the degree of FDG uptake.

The observers were given notice that the lesions were FDG-positive thymic epithelial lesions ; however, they were blind to the following information : final pathology, staging, age, gender, clinical symptoms, laboratory data and indication for FDG-PET/ CT examination. On the FDG-PET images, the degree of FDG uptake and the presence of FDG-positive lymph nodes, dissemination, distant metastasis and other primary lesions were evaluated. On the CT images, the observers reviewed the size, contour, shape, contrast enhancement pattern and existence of cystic or necrotic portions, calcification, invasion of adjacent structures, dissemination, pleural effusion, pericardial effusion or lymphadenopathy of each lesion.

\section{Statistical analysis}

The difference between the SUVmax and size of each lesion were evaluated between subgroups using the Kruskal-Wallis test or t-test. A $p$ value less than 0.05 was considered to be statistically significant.

\section{RESULTS}

A summary is shown in Table 5 and images of type A, type B2 and thymic carcinomas are shown in Figures 1-3.

Table 5 : A summary of Tokushima University Hospital

\begin{tabular}{cccc}
\hline & LR & HR & CA \\
\hline Masaoka stage & & & \\
I & 7 & 2 & 0 \\
II & 3 & 2 & 1 \\
III & 1 & 3 & 4 \\
IVa & 0 & 6 & 0 \\
IVb & 0 & 0 & 6
\end{tabular}

SUVmax

$$
\text { mean (SD) } \quad 3.7(1.8) \quad 4.0(2.8) \quad 7.6(3.7)
$$

size $\mathrm{mm}$

$$
\text { mean (SD) } \quad 44(19) \quad 60(34) \quad 79(32)
$$

surface

$\begin{array}{rccc}\text { smooth } & 10 & 5 & 3 \\ \text { irregular } & 1 & 8 & 8\end{array}$

shape

$\begin{array}{rlll}\text { oval/round } & 7 & 3 & 2 \\ \text { lobulated } & 4 & 9 & 9 \\ \text { plaque } & 0 & 1 & 0\end{array}$

degeneratice change/necrosis

$\begin{array}{cccc}+ & 6 & 8 & 11 \\ - & 5 & 5 & 0\end{array}$

calcification

$\begin{array}{cccc}+ & 0 & 7 & 4 \\ - & 11 & 6 & 7\end{array}$

enhancement pattern on CT

$\begin{array}{cccc}\text { heterogeneous } & 4 & 6 & 11 \\ \text { homogeneous } & 4 & 5 & 0\end{array}$

degree of enhancement

\begin{tabular}{rccc} 
strong & 2 & 1 & 5 \\
slight-moderate & 6 & 10 & 6 \\
\hline
\end{tabular}

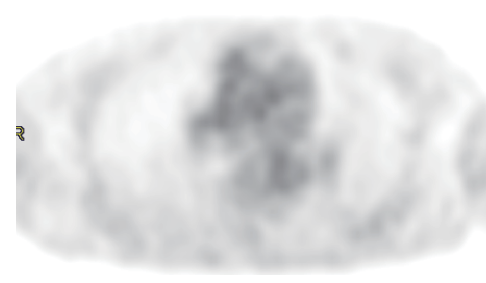

(a)

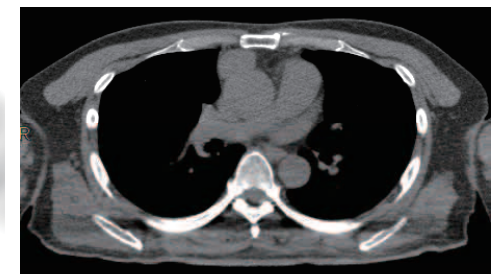

(b)

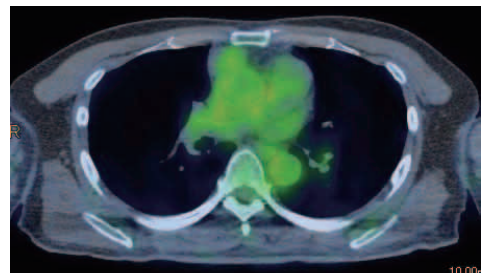

(c)

Fig 1 : Thymoma type A, Masaoka stage I. (a) FDG-PET, (b) CT, (c) FDG-PET/CT. The lesion measures 34 mm and is oval-shaped with a smooth surface. No necrosis or calcification are present. The SUVmax of the lesion is 2.7. 


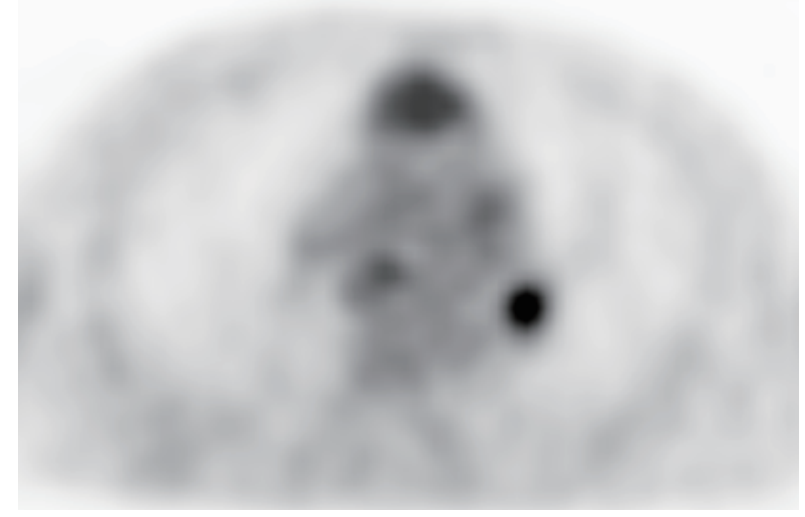

(a)

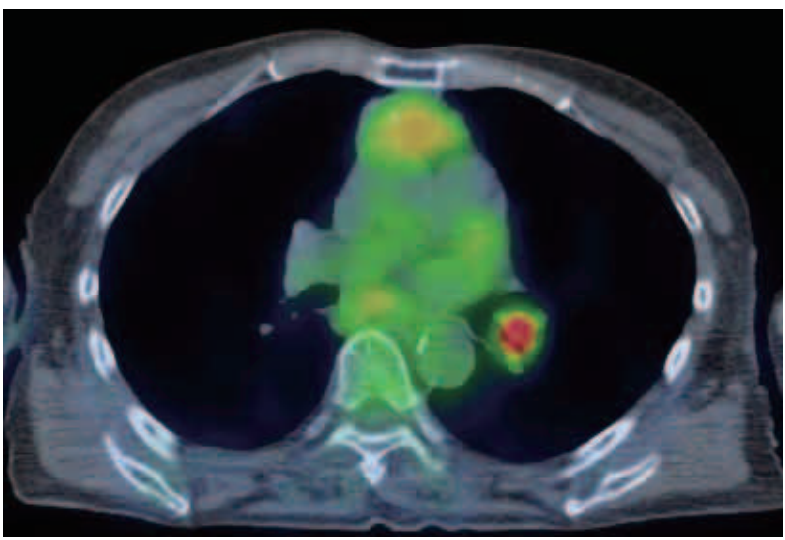

(c)

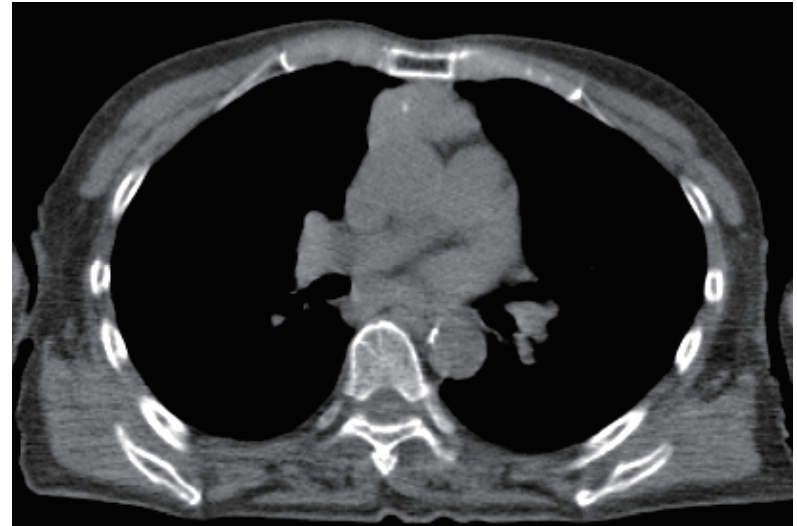

(b)

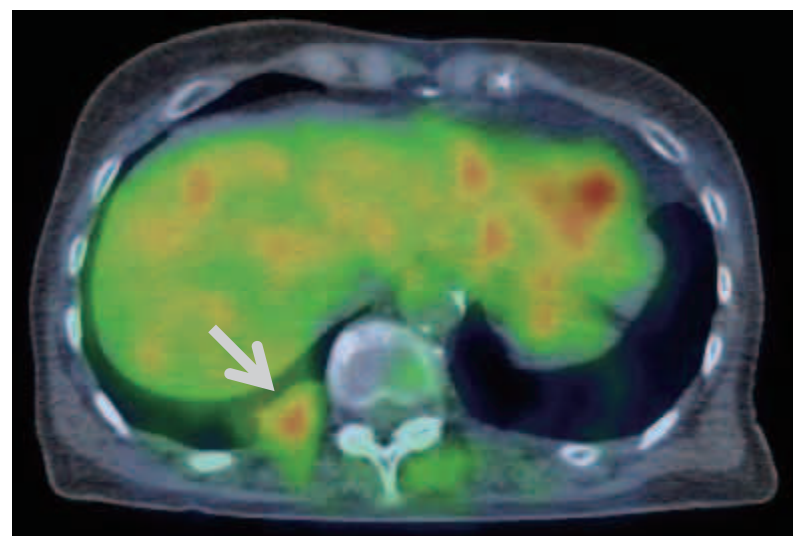

(d)

Fig 2 : Thymoma type B2, Masaoka stage IVa. (a) FDG-PET, (b) CT, (c) FDG-PET/CT, (d) FDG-PET/CT. The lesion measures $41 \mathrm{~mm}$ and is lobulated with an irregular surface. No necrosis is present. Spotty calcification is observed. The SUVmax of the lesion is 3.3. Pleural dissemination is also demonstrated (arrow in (d)). A reactive left hilar lymph node also shows FDG positive (a, c).

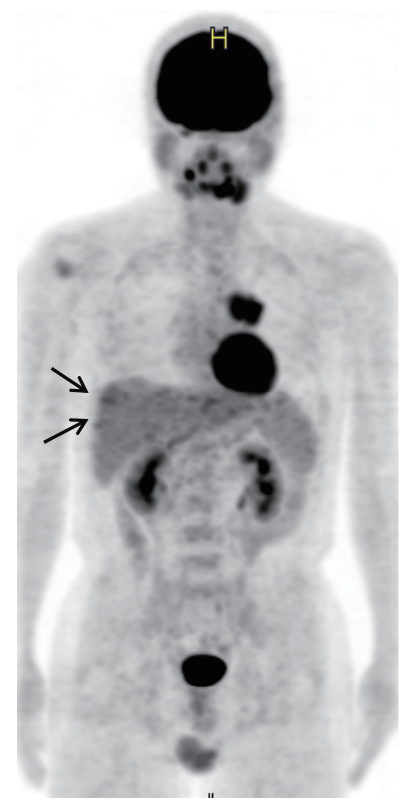

(a)

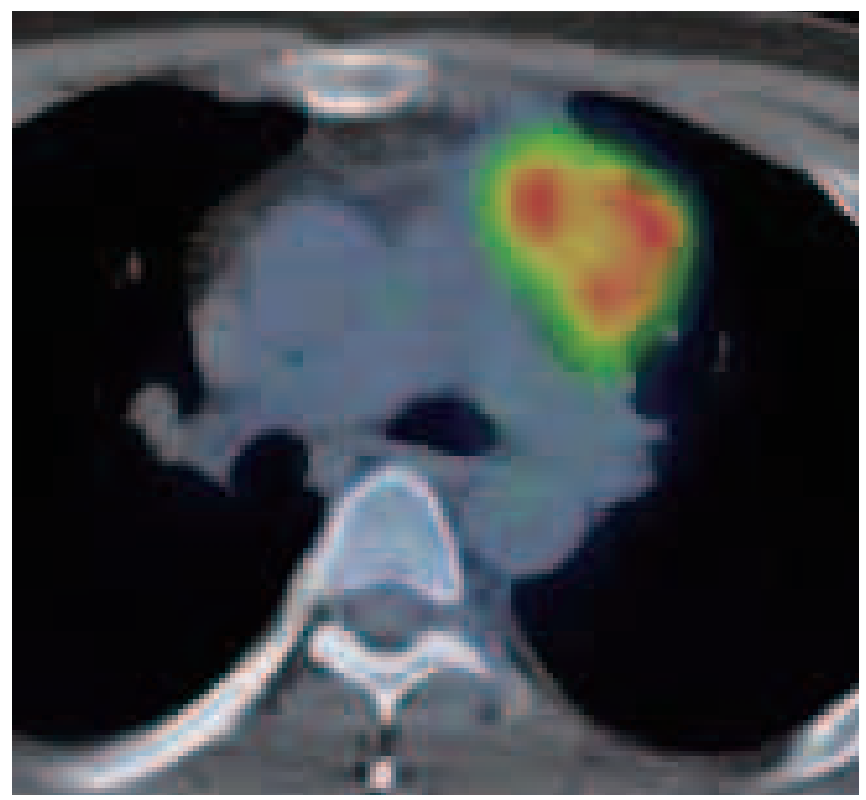

(b)

Fig 3 : Thymic carcinoma, Masaoka stage IVb. (a) MIP (maximum intensity projection) image of FDG-PET, (b) FDG-PET/CT. The lesion measures $57 \mathrm{~mm}$ and is lobulated with an irregular surface and necrosis. The lesion shows heterogeneous FDG uptake with SUVmax 6.3. Liver metastases are also demonstrated (arrow in (a)). 
Fifteen patients showed symptoms of chest pain or discomfort and two patients had blepharoptosis. Twenty patients were incidentally found to have abnormal anterior mediastinal lesions on chest Xray or chest CT. Pathologically, LR lesions were found in 11 patients (type $A$ : three patients, type $\mathrm{AB}$ : three patients and type $\mathrm{B} 1$ : five patients), HR lesions were found in 13 patients (type B1/2: two patients, type $\mathrm{B} 2$ : seven patients, type $\mathrm{B} 2 / 3$ : two patients and type B3 : two patients) and CAs were found in 11 patients. Two patients in the LR group had lung adenocarcinomas, one patient in the HR group had a large-cell lung carcinoma and one CA patient had FDG-negative ground glass opacity on CT that was confirmed to be a bronchoalveolar cell carcinoma using video-assisted thoracic surgery. Ten of 11 LR patients (91\%) were found to be in early Masaoka stages (I, II), whereas 10 of $11 \mathrm{CA}$ patients (91\%) were found to be in advanced stages (III, IV). Six of 11 (55\%) CA patients were found to have metastasis at the initial diagnosis. The Masaoka stages of the HR lesions varied from early to advanced, with advanced stages dominating. Nine of 13 patients $(70 \%)$ were diagnosed in stages III or IV.

The SUVmax values of the CA lesions were found to be higher than those of the LR and HR lesions (mean +/-SD : CA : 7.6 +/- 3.7, LR : $3.7+/-1.8$ and HR : $4.0+/-2.3)$. The differences between the values of the CA and LR lesions and the CA and HR lesions were statistically significant $(p=0.007$ and 0.008 , respectively), while the difference between the values of the HR and LR lesions was not significant $(p=0.99)$. The difference between the values of the thymoma (LR and HR) and thymic carcinoma lesions was statistically significant $(p=0.000482)$. The LR lesions tended to be smaller and the CA lesions tended to be larger with a statistically significant difference $(p=0.022)$. The LR lesions had round/oval shapes with smooth margins, while the CA lesions were lobulated with irregular margins. The HR lesions had various shapes and margins and tended to be lobulated with irregular margins. Degenerative changes and necrosis were found in all CA lesions and in six of 11 (55\%) LR lesions and eight of 13 (62\%) HR lesions. Calcification was detected in $31 \%$ of all lesions $(11 / 35)$. No calcification was detected in the LR lesions. The CA lesions showed heterogeneous contrast enhancement on $\mathrm{CT}$, and the enhancement pattern varied in the LR and HR lesions. The levels of anti-acetylcholine receptor (anti-AchR) antibodies were analyzed in a limited number of patients. Two of 10 (20\%) LR patients, one of nine (11\%) HR patients and zero CA patients were found to have elevated values. One HR patient with an elevated anti-AchR level had symptoms of myasthenia gravis.

\section{DISCUSSION}

The prognoses of patients with thymic epithelial lesions are significantly correlated with two factors : histological classification and clinical staging. The WHO updated the histological classification to include five subtypes of thymomas and thymic carcinomas in 2004. It is reported that thymic epithelial tumors found that the 5 -year survival rate was $100 \%$ in patients with type A tumors, $93 \%$ in patients with type $\mathrm{AB}$ tumors, $89 \%$ in patients with type $\mathrm{B} 1$ tumors, $82 \%$ in patients with type $\mathrm{B} 2$ tumors, $71 \%$ in patients with type B3 tumors and $48.0 \%$ in patients with thymic carcinomas (8). Therefore, patients with low-risk thymomas have good prognoses, whereas the prognoses of patients with high-risk thymomas worsen with the progression from type B2 to type B3 lesions and patients with thymic carcinomas have poor prognoses. Masaoka's criteria is widely used to determine clinical staging. The criteria are based on the presence of invasion in adjacent mediastinal adipose tissue or surrounding organs, and Masaoka staging significantly correlates with prognosis. The 20 -year survival rate is $89 \%$ in patients with stage I disease, $91 \%$ in patients with stage II disease, $49 \%$ in patients with stage III disease and $0 \%$ in patients with stage VIa or VIb disease. There is a significant divergence in prognosis between patients with early (stages I, II) and advanced stage (stages III, IV) disease. Therefore, predicting whether the histological grade is low-risk, high-risk or carcinoma and properly diagnosing the existence of invasion to surrounding organs such as the pericardium, great vessels or lungs are important before performing invasive procedures or administrating treatment. CT and MRI are reported to be useful in evaluating thymic epithelial lesions (9-12). On CT, thymomas appear as round or oval-shaped soft tissue masses with mild to moderate contrast enhancement. Degenerative changes or necrosis present as low density areas in the lesions. Calcification is detected by the presence of high density spots. Jeong reported that, on CT, lobulated contours were more often seen in high-risk thymomas and thymic carcinomas (9). Sadohara reported that the presence of irregular contours, necrosis or cystic components, 
heterogeneous enhancement and lymphadenopathy are highly suggestive of thymic carcinomas (10). In our study, the LR lesions tended to be smaller than the CA lesions with a statistically significant difference $(p=0.005)$. The LR lesions were round/ oval-shaped with smooth margins, while the CA lesions were lobulated with irregular margins. The HR lesions had various shapes and margins and tended to be lobulated with irregular margins. Degenerative changes and necrosis were found in all CA lesions and in $50 \%$ of the LR lesions and seven of $11(64 \%)$ of the HR lesions. Calcification was detected in $29 \%$ of all lesions (9/31). No calcification was detected in the LR lesions. The CA lesions showed heterogeneous contrast enhancement on $\mathrm{CT}$, while the enhancement pattern varied in the LR and HR lesions. On MRI, thymomas show low intensity on T1-weighted images, similar to that of muscle, and relatively high intensity on $\mathrm{T} 2$-weighted images. T2-weighted images show necrosis as high intensity and internal septa as low intensity. T1weighted images show various signal patterns in cystic regions ranging from low to high intensity, reflecting the protein content and the presence of hemorrhage. It is reported that MRI can differentiate low-risk thymomas from high-risk thymomas by depicting contours, capsules, septa and contrast enhancement patterns. With respect to depicting invasion outside of capsules, MRI is reported to be superior to CT. Pathologically proving invasion in surgical specimens is the most reliable method of determining the presence of invasion, as CT and MRI cannot detect microscopic invasion. In this study, we underdiagnosed one thymic carcinoma patient.

FDG-PET/CT is an advancing modality used to evaluate tumor activity, glucose metabolism and whole body morphology in a single session. It has become an important noninvasive diagnostic tool in the management of thymic lesions. FDG accumulates in tumor cells according to viability or pathological grading. Moreover, FDG accumulation is affected by various factors, such as glucose metabolism, hypoxia, angiogenesis. FDG accumulation of low-risk thymoma is low, and FDG accumulation of thymic carcinoma is high. High-risk thymoma exhibits various degrees of accumulation and a definite conclusion has not yet been reached; however, overall, the higher the histological grade, the higher the FDG accumulation becomes, suggesting that this is correlated to staging. FDG-PET may indicate lymph node metastasis and distant metastasis, which are sometimes difficult to detect using other modalities. When lymph node metastasis and/or distant metastasis are suspected in addition to thymic lesions, it is not practical to histologically confirm the metastatic lesions, and in such cases, FDG-PET/ $\mathrm{CT}$, which allows for searching of the entire body at once, is useful.

In summary, FDG-PET/CT is effective for estimating histopathological malignancy and staging in the diagnosis of thymic epithelial tumors and may be an important imaging test with high relevance to the prognosis of a patient.

Some portions of the data were presented at the 58 th annual meeting of the Society of Nuclear Medicine in 2011. The author greatly thanks "the Nuclear Medicine Team" of Tokushima University Hospital.

\section{REFERENCES}

1. Sung YM, Lee KS, Kim BT, Choi JY, Shim YM, Yi CA $:{ }^{18} \mathrm{~F}$-FDG PET/CT of thymic epithelial tumors : usefulness for distinguishing and staging tumor subgroups. J Nucl Med 47(10) : 1628-34, 2006

2. Endo M, Nakagawa K, Ohde Y, Okumura T, Kondo H, Igawa S, Nakamura Y, Tsuya A, Murakami H, Takahashi T, Yamamoto N, Ito I, Kameya T : Utility of 18FDG-PET for differentiating the grade of malignancy in thymic epithelial tumors. Lung Cancer 61(3) : 350-5, 2008

3. Inoue A, Tomiyama N, Tatsumi M, Ikeda $\mathrm{N}$, Okumura M, Shiono H, Inoue M, Higuchi I, Aozasa K, Johkoh T, Nakamura H, Hatazawa $\mathrm{J}:{ }^{18} \mathrm{~F}$-FDG PET for the evaluation of thymic epithelial tumors : Correlation with the World Health Organization classification in addition to dual-time-point imaging. Eur J Nucl Med Mol Imaging 36(8) : 1219-25, 2009

4. Kaira K, Endo M, Abe M, Nakagawa K, Ohde Y, Okumura T, Takahashi T, Murakami H, Tsuya A, Nakamura Y, Naito T, Hayashi I, Serizawa M, Koh Y, Hanaoka H, Tominaga H, Oriuchi N, Kondo H, Nakajima T, Yamamoto $\mathrm{N}$ : Biologic correlation of $2-\left[{ }^{18} \mathrm{~F}\right]$-fluoro-2-deoxy-D-glucose uptake on positron emission tomography in thymic epithelial tumors. J Clin Oncol 28(23) : 3746-53, 2010

5. Nakajo M, Kajiya Y, Tani A, Yoneda S, 
Shirahama H, Higashi M, Nakajo M $:{ }^{18} \mathrm{FDG}$ PET for grading malignancy in thymic epithelial tumors : significant differences in ${ }^{18} \mathrm{FDG}$ uptake and expression of glucose transporter-1 and hexokinase II between low and high-risk tumors : preliminary study. Eur J Radiol 81(1) : 146-51, 2010

6. Igai H, Matsuura N, Tarumi S, Chang SS, Misaki N, Go T, Ishikawa S, Yokomise H : Usefulness of $\left[{ }^{18} \mathrm{~F}\right]$ fluoro-2-deoxy-D-glucose positron emission tomography for predicting the World Health Organization malignancy grade of thymic epithelial tumors. Eur J Cardiothorac Surg 40(1) : 143-5, 2011

7. Terzi A, Bertolaccini L, Rizzardi G, Luzzi L, Bianchi A, Campione A, Comino A, Biggi A : Usefulness of 18-F FDG PET/CT in the pretreatment evaluation of thymic epithelial neoplasms. Lung Cancer 74(2) : 239-43, 2011

8. Park MS, Chung KY, Kim KD, Yang WI, Chung JH, Kim YS, Chang J, Kim JH, Kim SK, Kim SK : Prognosis of thymic epithelial tumors according to the new World Health Organization histologic classification. Ann Thorac Surg 78 (3) : 992-7, 2004

9. Han J, Lee KS, Yi CA, Kim TS, Shim YM, Kim J, Kim K, Kwon OJ : Thymic epithelial tumors classified according to a newly established WHO scheme : CT and MR findings. Korean J Radiol 4(1) : 46-53, 2003

10. Sadohara J, Fujimoto K, Müller NL, Kato S, Takamori S, Ohkuma K, Terasaki H, Hayabuchi $\mathrm{N}$ : Thymic epithelial tumors : comparison of CT and MR imaging findings of low-risk thymomas, high-risk thymomas, and thymic carcinomas. Eur J Radiol 60(1) : 70-9, 2006

11. Tomiyama $\mathrm{N}$, Honda $\mathrm{O}$, Tsubamoto $\mathrm{M}$, Inoue A, Sumikawa H, Kuriyama K, Kusumoto M, Johkoh T, Nakamura H : Anterior mediastinal tumors : diagnostic accuracy of CT and MRI. Eur J Radiol 69(2) : 280-8, 2009

12. Takahashi K, Al-Janabi NJ : Computed tomography and magnetic resonance imaging of mediastinal tumors. J Magn Reson Imaging 32(6) : 1325-39, 2010 